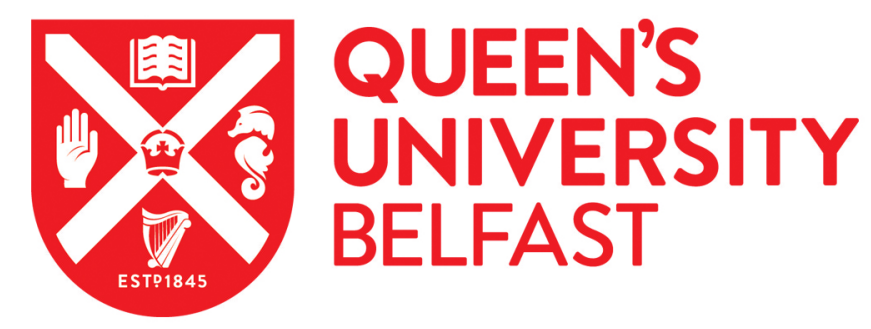

\title{
Extracellular Vesicle Biogenesis in Helminths: More than One Route to the Surface?
}

de la Torre Escudero, E., Bennett, A. P. S., Clarke, A., Brennan, G. P., \& Robinson, M. W. (2016). Extracellular Vesicle Biogenesis in Helminths: More than One Route to the Surface? Trends in Parasitology, 32(12), 921-929. https://doi.org/10.1016/j.pt.2016.09.001

\section{Published in:}

Trends in Parasitology

Document Version:

Peer reviewed version

Queen's University Belfast - Research Portal:

Link to publication record in Queen's University Belfast Research Portal

Publisher rights

(C) 2016 Elsevier Ltd.

This manuscript version is made available under the CC-BY-NC-ND 4.0 license http://creativecommons.org/licenses/by-nc-nd/4.0/,which permits distribution and reproduction for non-commercial purposes, provided the author and source are cited.

\section{General rights}

Copyright for the publications made accessible via the Queen's University Belfast Research Portal is retained by the author(s) and / or other copyright owners and it is a condition of accessing these publications that users recognise and abide by the legal requirements associated with these rights.

Take down policy

The Research Portal is Queen's institutional repository that provides access to Queen's research output. Every effort has been made to ensure that content in the Research Portal does not infringe any person's rights, or applicable UK laws. If you discover content in the Research Portal that you believe breaches copyright or violates any law, please contact openaccess@qub.ac.uk. 
Eduardo de la Torre-Escudero, Adam P.S. Bennett, Alexzandra Clarke, Gerard P. Brennan and Mark W. Robinson* Belfast, 97 Lisburn Road, Belfast, Northern Ireland.

*Correspondence: mark.robinson@qub.ac.uk (M.W. Robinson).

Keywords: helminth, exosome, extracellular vesicle, microvesicle, bleb

\section{Abstract}

The quite recent discovery that parasites release extracellular vesicles (EVs) that can transfer a range of effector molecules to host cells has made us re-think our understanding of the hostparasite interface. In this opinion article we will consider how recent proteomics and transcriptomics studies, together with ultrastructural observations, suggest that more than one mechanism of EV biogenesis can occur in helminths. We propose that distinct EV sub-types have roles in immune-modulation and repair of drug-induced damage, and put forward the case for targeting EV biogenesis pathways to achieve parasite control. In doing so we raise a number of outstanding research questions that must be addressed before this can happen. 
The persistence of helminth parasites in their mammalian hosts has been ascribed to their striking ability to manipulate host immune responses. Many helminths are obligate blood feeders and can deliver secreted molecules into the bloodstream where they exert an immunosuppressive activity on the host immune system [1]. Other molecules are secreted from the gut, excretory pores and surface cuticle/tegument into the local microenvironment, often the host intestine [2-4]. For many years the paucity of material made these secretions extremely difficult to analyse but the application of mass spectrometry-based proteomics techniques has allowed a detailed understanding of the type and abundance of the various proteins present in soluble helminth secretions (reviewed in [5]). However, until quite recently the contribution of extracellular vesicles (EVs; see glossary) to helminth secretomes has been overlooked.

EVs are small membrane-bound structures that are shed by most cell types. Although once considered to act solely as a cellular waste disposal system [6], EVs are now recognised as important mediators of cell-cell communication by transferring a range of effector molecules including proteins, lipids, mRNA, microRNA and other non-coding RNA species. Variously described as exosomes or microvesicles (MVs) depending on their composition, size and mode of biogenesis (see below), EVs not only perform a variety of roles in the maintenance of normal physiology but also participate in pathological settings, notably in tumour progression [7-9]. A growing number of studies have also shown that parasite-derived EVs play an important role during infection and pathogenesis [10]. Although most of these studies have focused on profiling the molecular cargo packaged into parasite EVs, they have provided a valuable first insight into the putative mechanism(s) used by parasites for EV 
47 biogenesis in mammalian cells and examine the ultrastructural and biochemical evidence for EV biogenesis and export mechanisms used by helminth parasites.

EV biogenesis pathways in mammalian cells

There are two major subtypes of EVs that are actively released by viable cells: exosomes and microvesicles (MVs). Exosomes are typically 30-100 nm in diameter and originate from the endosomal pathway of eukaryotic cells. The process starts with inward budding of the late endosome membrane which forms multivesicular bodies (MVBs) containing a number of intraluminal vesicles (ILVs). The ILVs are then secreted from the cell surface (as exosomes) following fusion of the MVB with the plasma membrane. The principal machinery that drives this process is the endosomal sorting complex required for transport (ESCRT). ESCRT-0, -I, and -II complexes cluster ubiquitinated proteins to the endosomal membrane, whilst ESCRT-III forms polymeric filaments that result in the invagination of the membrane, forming the ILV [11-14]. The final abscission of the budding ILVs into the MVB lumen requires the AAA-ATPase VPS4 [15]. However, ESCRT-independent pathways have been reported that centre around several members of the tetraspanin family [16] or that require the generation of ceramide by neutral sphingomyelinase [17]. In this respect, Colombo et al. [18] proposed four molecular machineries involved in ILV formation: (1) the ESCRT pathway; (2) the lipid pathway primarily involving the lipid hydrolases neutral sphingomyelinase and phospholipase D2; (3) the tetraspanins route where CD63 plays a principal role; and (4) a hybrid mechanism combining elements of all the former machineries together. The subsequent fusion of MVBs with the plasma membrane is dependent on small GTPases including Ral-1, Rab8b, Rab11, Rab27a and Rab27b and the Rab effectors otoferlin and synaptotagmin-like protein [19-22]. 
by direct budding from the plasma membrane in response to a variety of external stimuli

which generally lead to elevated intracellular $\mathrm{Ca}^{2+}$ levels and cytoskeleton-driven membrane remodelling $[23,24]$. This $\mathrm{Ca}^{2+}$ signalling cascade activates scramblase that is responsible for the translocation of phosphatidylserine to the outer leaflet of the plasma membrane. The resulting membrane asymmetry is thought to drive localised curvature of the plasma membrane. MV budding is driven further by the rearrangement of the actin cytoskeleton conducted by calpain [25]. Many studies suggest that small GTPases, such as ARF6, play a key role in MV formation by indirectly activating proteases in response to the activation of the extracellular signal-regulated kinase (ERK) [24]. Although the final abscission mechanism of the MV is not fully understood, the late ESCRT component VPS4 is likely to be involved in the same way that it drives abscission of the budding ILVs into the MVB lumen during exosome biogenesis [26]. Whilst both exosomes and MVs can deliver cargo to target cells, and are released by viable cells in an energy-dependent manner, most cells constitutively produce exosomes whilst MVs are generally released in response to specific signals [27].

\section{EVs as potential targets for anti-parasite therapy}

Several studies have described the modulatory effects of parasite-derived EVs on the host immune system [28-30] or the pathological effects on host cells [31]. Indeed, the role of helminth EVs as vehicles for transfer of small RNAs (especially miRNA) to host cells has been extensively documented in the last few years [28,32-35] suggesting a new mechanism used by parasites to influence host cell function at a molecular level. Targeting key regulators of EV biogenesis (including sphingomyelinase, ESCRT components and Rab GTPases) using chemical inhibitors or RNAi has been shown to significantly reduce the number of EVs 
released by mammalian cells (Table 1). Indeed, suppressing EV production to inhibit their effect in disease is an emerging therapeutic strategy that has yielded impressive results, notably in cancer therapy (reviewed in [8]). In the same vein, parasite EV biogenesis pathways might be an attractive therapeutic target if selective inhibitors could be identified. Disrupting the packaging, biogenesis and/or release of parasite EVs would prevent the delivery of a plethora of protein or small RNA immunomodulators to host cells; thus, allowing the host to mount an effective immune response against the parasite. Silencing of a range of orthologues of molecules that function at different stages of EV biogenesis pathways in mammalians cells led to varied aberrant phenotypes in Caenorhabditis elegans (Table 1). Whilst the direct impact on EV production was not assessed for all of these targets, MVBs accumulated below the cuticle layer (and did not fuse with the plasma membrane) when the vATPase V0 subunit and GTPase Ral-1 were mutated or silenced $[22,45]$. The challenge now is to determine how silencing EV biogenesis components in parasitic helminths impacts their ability to establish infection and avoid the host immune response. For instance, silencing syntenin (involved in ILV formation and cargo sorting) had no effect on the ability of schistosomulae and adult schistosomes to survive in vitro culture however their ability to persist in mammalian hosts was not investigated [46].

\section{Exosome biogenesis in helminths - insights from Fasciola hepatica}

To date, much of our understanding of EV biogenesis in helminths comes from studies on the liver fluke, Fasciola hepatica. A detailed proteomics analysis of its secretome showed that $F$. hepatica releases at least two major sub-populations of EVs that differ according to size, cargo molecules and probable site of origin [47]. The sub-population of smaller EVs (30-100 nm diameter) contain a number of well-known exosomal markers, also identified in other helminth parasites, such as Hsp70, ALIX, tetraspanin CD63 and several Rab GTPases 
suggesting that Fasciola secretes bona fide exosomes. In support of this, mass spectrometry data strongly suggests an endosomal origin of the smaller secreted vesicles; up to ten proteins from the ESCRT pathway were found associated with the vesicles. These included members of the ESCRT-III complex and the Vps4 ATPase responsible for ILV budding and abscission respectively as well as the ESCRT-associated proteins ALIX and syntenin [47]. Compared with the plasma membrane, exosomal membranes are enriched in certain phospholipids as well as cholesterol and ceramide. They also contain numerous lipid-binding proteins such as annexins that are organized in specialised lipid rafts called tetraspaninenriched microdomains (TEMs). Lipid hydrolases (typically sphingomyelinases and phospholipase B) and cholesterol transporters are also characteristic features of exosome membranes [9]. TEMs are believed to have roles in both exosome biogenesis and the sorting of cargo by promoting specific compartmentalisation of proteins and receptor molecules into the budding ILVs [16]. According to the mass spectrometry data, the $F$. hepatica exosomes contain all the necessary proteins to organise the membrane into TEMs (including several members of the tetraspanin and annexin families, an acid sphingomyelinase, a phospholipase B2-like enzyme as well as the cholesterol transporters Niemann Pick C1 and C2 proteins; [47]). Due to their biophysical properties, TEMs have also been defined as detergent-resistant microdomains [48]. Such organization would confer a high rigidity to $F$. hepatica EV membranes (and presumably to those from other helminths) at neutral $\mathrm{pH}$ that would help to preserve their structural integrity, and persistence, in host bile and other biological fluids. Indeed, exosome-like vesicles from the nematode parasite Brugia malayi have been detected in host circulatory blood, suggesting that they may act at effector sites well beyond that of the local host microenvironment [30]. Recently it was shown that exosome membranes from the nematode parasite Heligmosomoides polygyrus are enriched with plasmalogens and other 
derived exosomes [49]. Thus, is it likely that the unique lipid and protein composition of parasite EV membranes has evolved to confer the greatest protection possible to the cargo packaged within the vesicle lumen.

\section{Ultrastructural evidence for EV biogenesis in helminths}

Whilst much of the biochemical and proteomics data to date supports an endosomal origin for helminth exosomes, further ultrastructural studies are required to confirm this. Exosome-like vesicles have been described associated with the lining of the gut in Schistosoma mansoni and in $H$. polygyrus $[28,50]$. Secretion of exosomes from the cuticle surface in parasitic nematodes has yet to be documented although exosomes released by $C$. elegans epithelial cells are more likely to contribute to formation of the overlaying cuticle rather than be secreted into the extracellular environment from the worm surface [22].

Transmission electron microscopy has revealed the presence of structures within the tegumental syncytium of parasitic trematodes that resemble the MVBs observed in mammalian cells (Figure 1) $[4,32,51]$. These structures have been shown to fuse with the apical plasma membrane at the tegumental surface from where vesicles, compatible in size with exosomes, are discharged [51]. These observations, and the fact that trematode exosomes contain a considerable number of tegumental-associated proteins [47], suggest a role for the tegument in exosome secretion. It is noteworthy, given the multitude of ultrastructural studies performed on $F$. hepatica and other trematodes, that these putative MVBs have not been described more widely in the literature. Wilson et al. [4] reported the appearance of MVB-like structures within the $F$. hepatica tegumental syncytium in response to culturing the flukes in vitro which could suggest that some helminth EVs are released in response to stress. Indeed, the transient nature of MVB formation and exosome release makes 
the phenomenon difficult to observe (even in mammalian cells) [18], but it could also indicate that alternative mechanisms of EV release are in operation in the parasite.

\section{Are there novel EV biogenesis pathways in helminths?}

Several studies have suggested the existence of atypical EV biogenesis pathways that combine elements of the ESCRT, lipid and tetraspanin pathways in mammalian cells [18]. Whilst the proteomics data largely supports an ESCRT-dependent origin for the exosome-like EVs released by $F$. hepatica, we also identified some components of ESCRT-independent pathways, such as sphingomyelinase and various members of the tetraspanin family, in its secretome [47]. Whilst this may be due to the presence of several sub-populations of EVs (either produced by distinct mechanisms or released from different parasite tissues), it could also indicate the coordinated participation of proteins from ESCRT-dependent and independent pathways in the production of exosome-like vesicles in $F$. hepatica, as has been described in other cell types [52]. In contrast, the larger Fasciola EVs were shown to be specifically enriched with the inactive $37 \mathrm{kDa}$ cathepsin $\mathrm{L}$ zymogen and may originate from the specialised secretory cells that line the parasite gastrodermus [47]. Our preliminary biochemical characterisation of these EVs suggest they are distinct from the smaller exosome-like vesicles with respect to their cargo molecules. Data pertaining to their mechanism of biogenesis is currently lacking, although MVBs have not been described in the parasite gastrodermal cells. One possibility is the breakdown of the long finger-like membrane-bound lamellae, which extend from the gastrodermal cells into the gut lumen, to release free EVs. A similar mechanism has been described in the protozoan parasite, Trypanosoma brucei rhodesiense. These single-celled parasites produce membrane-bound nanotubes that extend up to $20 \mu \mathrm{m}$ from the cell that disassociate into free EVs of around 80 $\mathrm{nm}$ in diameter [53]. Further proteomics analysis of the gastrodermal cell-derived EVs and 
subsequent immunolocalisation studies are required to investigate this possibility in fluke and other helminths.

\section{Are drug-induced "blebs" functionally equivalent to microvesicles?}

The appearance of membrane-bound "blebs" on the parasite surface is a common initial response to drug-induced stress that has been observed in various flatworm and roundworm parasites [54-56]. Blebs appear as vesicular structures that eventually pinch off from the plasma membrane bounding the parasite surface and have been best described by scanning electron microscopy studies of the trematode tegument. The released blebs are variable in size, usually ranging between $200 \mathrm{~nm}$ and a few micrometres, but can be more than $20 \mu \mathrm{m}$ in diameter. Complementary transmission electron microscopy studies have confirmed that the blebs pinch directly from the tegumental surface and are released into the extracellular environment (Figure 2) [55,57-59]. It has been suggested that blebbing is an attempt by helminths to replenish tegument that has been lost or damaged due to drug action [60] or humoral immune challenge [61]. Given the abundance of pumps such as P-glycoprotein 1 in the parasite EV membrane [47] it is possible that blebs sequester anthelmintics before their release; thus, reducing their effective local concentration in the parasite tissues. Indeed, Pglycoprotein reversal agents have been shown to increase the efficacy of triclabendazole (TCBZ) against TCBZ-resistant $F$. hepatica [62].

The mechanism of bleb formation in helminths has yet to be determined, but it is thought to be a calcium-dependent process $[63,64]$. MVs also bud directly from the plasma membrane in response to raised intracellular $\mathrm{Ca}^{2+}$ levels [65]. Given these biochemical and structural similarities, it is conceivable that parasite blebs are formed using the same molecular machinery as the MVs released by mammalian cells; i.e. $\mathrm{Ca}^{2+}$-dependent cytoskeleton-driven remodelling of the plasma membrane. Transcriptomics and proteomics 
analysis indicates that the major players in this process (including ARF6 GTPase, pivotal in MV biogenesis in mammalian cells) are conserved in $F$. hepatica although functional studies, such as RNAi, are needed to confirm their role in MV/bleb formation [47].

\section{Concluding remarks}

The discovery of parasite-derived EVs has changed our view of the host-parasite interface. The formation of EVs enables the parasite to release a range of effector molecules (including degradation-susceptible protein and RNA species) in a protected manner to ensure their safe delivery to host cells. The cargo of helminth exosome-like EVs contain numerous immunomodulatory proteins and microRNAs that alter host immune cell phenotype to support parasite survival and reproduction [28,33,47]. Additionally, helminth blebs/MVs likely represent a vital membrane repair mechanism in response to drug-induced damage or host antibody attack. If the molecular machinery responsible for either of these EV biogenesis pathways could be disrupted using selective inhibitors/blocking antibodies, it might just be possible to tip the host immune balance in favour of parasite elimination; by preventing immune suppression by EV-delivered parasite immunomodulators or to enhance the potency of existing anthelmintic drugs by impairing parasite membrane repair mechanisms. Whilst recent proteomics and functional studies have considerably advanced our understanding of parasite EVs, many basic research questions remain (see Outstanding Questions). By addressing these areas, we may have a realistic chance of targeting EVs to achieve parasite control in the future.

\section{Glossary}

- Bleb: a blister-like protrusion of the plasma membrane on the cell surface. 
- Endosomal sorting complex required for transport (ESCRT): a series of up to 30 membrane-associated proteins that interact directly to form distinct molecular machineries that drive recruitment of cargo molecules, inward budding and abscission of the endosomal membrane during ILV or MVB formation.

- Extracellular vesicle: small membrane-bound vesicles shed from most viable cell types. Includes exosomes (30-100 $\mathrm{nm}$ in diameter) that are formed from the endosomal pathway and microvesicles (100-1000 nm in diameter) that pinch directly from the plasma membrane.

- Multivesicular body (MVB): a mature endosome that contains numerous vesicles (termed intra-luminal vesicles; ILVs) within their interior. They are formed by inward budding of the endosomal membrane followed by abscission of the ILV into the endosome lumen.

\section{Acknowledgements}

This work was supported by a grant to M.W.R. (BB/L019612/1) from the BBSRC. A.P.S.B. is supported by a postgraduate studentship from the Northern Ireland Department for Employment and Learning (DEL).

\section{References}

1. Molina-Hernández, V. et al. (2015) Fasciola hepatica vaccine: we may not be there yet but we're on the right road. Vet. Parasitol. 208, 101-111

2. Fairweather, I. et al. (1999) Development of Fasciola hepatica in the mammalian host. In Fasciolosis (Dalton, J.P., ed) pp. 1-29, CABI

3. Collins, P.R. et al. (2004) Cathepsin L1, the major protease involved in liver fluke (Fasciola hepatica) virulence: propeptide cleavage sites and autoactivation of the zymogen secreted from gastrodermal cells. J. Biol. Chem. 279, 17038-17046

4. Wilson, R.A. et al. (2011) Exploring the Fasciola hepatica tegument proteome. Int. J. Parasitol. 41, 1347-1359 
5. van der Ree, A.M. and Mutapi, F. (2015) The helminth parasite proteome at the hostparasite interface - informing diagnosis and control. Exp. Parasitol. 157, 48-58

6. Thébaud, B. and Stewart, D.J. (2012) Exosomes: cell garbage can, therapeutic carrier, or trojan horse? Circulation 126, 2553-2555

7. Yáñez-Mó, M. et al. (2015) Biological properties of extracellular vesicles and their physiological functions. J Extracell Vesicles 4:27066.

8. EL Andaloussi, S. et al. (2013) Extracellular vesicles: biology and emerging therapeutic opportunities. Nat. Rev. Drug. Discov. 12, 347-357

9. Record, M. et al. (2014) Exosomes as new vesicular lipid transporters involved in cell-cell communication and various pathophysiologies. Biochim. Biophys. Acta. $1841,108-120$

10. Coakley, G. et al. (2015) Exosomes and other extracellular vesicles: the new communicators in parasite infections. Trends Parasitol. 31, 477-89

11. Schorey, J.S. et al. (2014) Exosomes and other extracellular vesicles in host-pathogen interactions. EMBO Rep. 16, 24-43

12. Akers, J.C. et al. (2013) Biogenesis of extracellular vesicles (EV): exosomes, microvesicles, retrovirus-like vesicles, and apoptopic bodies. J. Neurooncol. 113, 111

13. Kowal, J. et al. (2014) Biogenesis and secretion of exosomes. Curr. Opin. Cell Biol. 29, 116-125

14. Simons, M. and Raposo, G. (2009) Exosomes - vesicular carriers for intercellular communication. Curr. Opin. Cell Biol. 21, 575-581

15. Hasegawa, T. et al. (2011) The AAA-ATPase VPS4 regulates extracellular secretion and lysosomal targeting of $\alpha$-synuclein. PLOS ONE 6, e29460

16. Andreu, Z. and Yáñez-Mó, M. (2014) Tetraspanins in extracellular vesicle formation and function. Front. Immunol. 4, 442

17. Trajkovic, K. et al. (2008) Ceramide triggers budding of exosome vesicles into multivesicular endosomes. Science 319, 1244-1247

18. Colombo, M. et al. (2014) Biogenesis, secretion, and intercellular interactions of exosomes and other extracellular vesicles. Annu. Rev. Cell Dev. Biol. 30, 255-89

19. Chen, S. et al. (2001) Rab8b and its interacting partner TRIP8b are involved in regulated secretion in AtT20 cells. J. Biol. Chem. 276, 13209-13216

20. Savina, A. et al. (2005) Rab11 promotes docking and fusion of multivesicular bodies in a calcium-dependent manner. Traffic 6, 131-143 
21. Ostrowski, M. et al. (2010) Rab27a and Rab27b control different steps of the exosome secretion pathway. Nat. Cell. Biol. 12, 19-30

22. Hyenne, V. et al. (2015) RAL-1 controls multivesicular body biogenesis and exosome secretion. J. Cell Biol. 211, 27-37

23. Hugel, B. et al. (2005) Membrane microparticles: two sides of the coin. Physiology (Bethesda) 20, 22-7

24. Muralidharan-Chari, V. et al. (2009) ARF6-regulated shedding of tumor cell-derived plasma membrane microvesicles. Curr. Biol. 19, 1875-85

25. Fox, J.E. et al. (1990) Role of the membrane skeleton in preventing the shedding of procoagulant-rich microvesicles from the platelet plasma membrane. J. Cell Biol. 111, 483-93

26. Booth, A.M. et al. (2006) Exosomes and HIV Gag bud from endosome-like domains of the T cell plasma membrane. J. Cell Biol. 172, 923-35

27. Angelot, F. et al. (2009) Endothelial cell-derived microparticles induce plasmacytoid dendritic cell maturation: potential implications in inflammatory diseases. Haematologica 94, 1502-12

28. Buck, A.H. et al. (2014) Exosomes secreted by nematode parasites transfer small RNAs to mammalian cells and modulate innate immunity. Nat. Commun. 5, 5488

29. Wang, L. et al. (2015) Exosome-like vesicles derived by Schistosoma japonicum adult worms mediates M1 type immune- activity of macrophage. Parasitol. Res. 114, $1865-1873$

30. Zamanian, M. et al. (2015) Release of small RNA-containing exosome-like vesicles from the human filarial parasite Brugia malayi. PLoS Negl. Trop. Dis. 9, e0004069

31. Chaiyadet, S. et al. (2015) Carcinogenic liver fluke secretes extracellular vesicles that promote cholangiocytes to adopt a tumorigenic phenotype. J. Infect. Dis. 212, 163645

32. Bernal, D. et al. (2014) Surface analysis of Dicrocoelium dendriticum. The molecular characterization of exosomes reveals the presence of miRNAs. J. Proteomics 105, 232-41

33. Hansen, E.P. et al. (2015) Secretion of RNA-containing extracellular vesicles by the porcine whipworm, Trichuris suis. J. Parasitol. 101, 336-40

34. Fromm, B. et al. (2015) The revised microRNA complement of Fasciola hepatica reveals a plethora of overlooked microRNAs and evidence for enrichment of immuno-regulatory microRNAs in extracellular vesicles. Int. J. Parasitol. 45, 697702 
35. Nowacki, F.C. et al. (2015) Protein and small non-coding RNA-enriched extracellular vesicles are released by the pathogenic blood fluke Schistosoma mansoni. J. Extracell. Vesicles 4, 28665

36. Colombo, M. et al. (2013) Analysis of ESCRT functions in exosome biogenesis, composition and secretion highlights the heterogeneity of extracellular vesicles. $J$. Cell Sci. 126, 5553-65

37. Baietti, M.F. et al. (2012) Syndecan-syntenin-ALIX regulates the biogenesis of exosomes. Nat. Cell Biol. 4, 677-85

38. Bianco, F. et al. (2009) Acid sphingomyelinase activity triggers microparticle release from glial cells. EMBO J. 28, 1043-54

39. Hsu, C. et al. (2010) Regulation of exosome secretion by Rab35 and its GTPaseactivating proteins TBC1D10A-C. J. Cell Biol. 189, 223-32

40. Beckett, K. et al. (2013) Drosophila S2 cells secrete wingless on exosome-like vesicles but the wingless gradient forms independently of exosomes. Traffic 14, 8296

41. Abrami, L. et al. (2013) Hijacking multivesicular bodies enables long-term and exosome-mediated long-distance action of anthrax toxin. Cell Rep. 5, 986-96

42. Koles, K. et al. (2012) Mechanism of evenness interrupted (Evi)-exosome release at synaptic boutons. J. Biol. Chem. 287, 16820-34

43. Gross, J.C. et al. (2012) Active Wnt proteins are secreted on exosomes. Nat. Cell Biol. 14, 1036-45

44. Ghossoub, R. et al. (2014) Syntenin-ALIX exosome biogenesis and budding into multivesicular bodies are controlled by ARF6 and PLD2. Nat. Commun. 5, 3477

45. Liégeois, S. et al. (2006) The V0-ATPase mediates apical secretion of exosomes containing Hedgehog-related proteins in Caenorhabditis elegans. J. Cell Biol. 173, 949-61

46. Figueiredo, B.C. et al. (2014) Schistosome syntenin partially protects vaccinated mice against Schistosoma mansoni infection. PLoS Negl. Trop. Dis. 8, e3107

47. Cwiklinski, K. et al. (2015) The extracellular vesicles of the helminth pathogen, Fasciola hepatica: biogenesis pathways and cargo molecules involved in parasite pathogenesis. Mol. Cell. Proteomics 14, 3258-73

48. de Gassart, A. et al. (2003) Lipid raft-associated protein sorting in exosomes. Blood $102,4336-44$

49. Simbari, F. et al. (2016) Plasmalogen enrichment in exosomes secreted by a nematode parasite versus those derived from its mouse host: implications for exosome stability and biology. J Extracell Vesicles 5, 30741 
50. Wilson, R.A. (2012) Proteomics at the schistosome-mammalian host interface: any prospects for diagnostics or vaccines? Parasitology 139, 1178-94

51. Marcilla, A. et al. (2012) Extracellular vesicles from parasitic helminths contain specific excretory/secretory proteins and are internalized in intestinal host cells. PLoS ONE 7, e45974

52. Vilette, D. et al. (2015) Efficient inhibition of infectious prions multiplication and release by targeting the exosomal pathway. Cell. Mol. Life Sci. 72, 4409-27

53. Szempruch A.J. et al. (2016) Extracellular vesicles from Trypanosoma brucei mediate virulence factor transfer and cause host anemia. Cell 164, 246-57

54. Apinhasmit, W. and Sobhon, P. (1996) Opisthorchis viverrini: effect of praziquantel on the adult tegument. Southeast Asian J. Trop. Med. Public Health 27, 304-11

55. Robinson, M.W. et al. (2003) The effect of the microtubule inhibitor tubulozole-C on the tegument of triclabendazole-susceptible and triclabendazole-resistant Fasciola hepatica. Parasitol. Res. 91, 117-29

56. Zeng, X. et al. (2013) Angiostrongylus cantonensis: tegumental and hypodermic alterations of the fourth-stage larvae following administration of tribendimidine in vivo and in vitro. Parasitol. Res. 112, 3035-3040

57. Halferty, L. et al. (2009) Electron microscopical study to assess the in vitro effects of the synthetic trioxolane OZ78 against the liver fluke, Fasciola hepatica. Parasitology $136,1325-37$

58. Toner, E. et al. (2010) Tegumental surface changes in adult Fasciola hepatica in response to treatment in vivo with triclabendazole in the sheep host. Vet. Parasitol. $172,238-48$

59. Tansatit, T. et al. (2012) Fasciola gigantica: the in vitro effects of artesunate as compared to triclabendazole on the 3-weeks-old juvenile. Exp. Parasitol. 131, 8-19

60. Bennett, C.E. et al. (1980) Fasciola hepatica: changes in tegument during killing of adult flukes surgically transferred to sensitized rats. Parasite Immunol. 2, 39-55

61. Abdeen, S.H. et al. (2012) Ultrastructural changes of adult Schistosoma mansoni worms recovered from C57BL/6 mice passively immunized with normal and vaccinated rabbit sera in vivo. Parasitol. Res. 110, 37-47

62. Mottier, L. et al. (2006) Resistance-induced changes in triclabendazole transport in Fasciola hepatica: ivermectin reversal effect. J. Parasitol. 6, 1355-1360

63. Bricker, C.S. et al. (1983) The relationship between tegumental disruption and muscle contraction in Schistosoma mansoni exposed to various compounds. Z. Parasitenkd. $69,61-71$ 
64. Xiao, S. et al. (1984) Praziquantel-induced vesicle formation in the tegument of male

65. Cocucci, E. et al. (2009) Shedding microvesicles: artefacts no more. Trends Cell Biol. $19,43-51$ 

trematodes.

(A) Schematic representation of the proposed exosome biogenesis pathway in the trematode tegument and the major components likely involved based upon mass spectrometry analysis of secreted exosome-like extracellular vesicles (EVs). Endosomal sorting complexes required for transport (ESCRT)-dependent (TSG101, ALIX and VPS4) and -independent (aSMase and CD63 tetraspanin) components may contribute to the initial formation of multivesicular bodies (MVBs), which are directed towards the apical plasma membrane (APM) of the tegumental syncytium. Here, soluble N-ethylmaleimide-sensitive factor activating protein receptor (SNAREs) (such as syntaxin, SNAP-25 and VAMP7) together with small GTPases (including Rab11, Rab27 and Ral-A) may facilitate the tethering and fusion of the MVB to the plasma membrane allowing the release of parasite-derived exosomes into the host microenvironment. S, spine. (B) Transmission electron micrograph of the tegumental syncytium of adult Fasciola hepatica containing structures that resemble MVBs. Inset, magnification of a MVB-like structure containing several intraluminal vesicles (ILVs). aSMase, acid sphingomyelinase.

Figure 2. Microvesicle/bleb release from the apical plasma membrane of the trematode tegument.

(A) Schematic representation of the proposed mechanism of bleb/microvesicle (MV) formation and release from the apical plasma membrane (APM) of the trematode tegument. An increase in intracellular $\mathrm{Ca}^{2+}$ concentration triggers a signalling cascade that promotes the excision of MVs/blebs directly from the tegmental surface. This "budding" process requires a state of membrane asymmetry, conducted by phospholipid translocases (e.g. scramblase), which is driven further by calpain-dependent cleavage and rearrangement of the actin 

cytoskeleton. The actin-binding proteins ezrin, radixin and moesin (ERM) are also involved 507 in the reassembly of the cytoskeleton following MV/bleb formation. S, spine. (B) Scanning 508 electron micrograph of the surface of the Fasciola hepatica tegument showing profuse 509 blebbing. (C) Transmission electron micrograph of $F$. hepatica tegumental syncytium 510 showing the pinching of blebs/MVs from the apical plasma membrane and their release from 511 the tegumental surface. Arrowheads; MV/blebs. 
Table 1. Key regulators of EV biogenesis in mammalian cells and their orthologues in helminths

\begin{tabular}{|c|c|c|c|c|c|c|}
\hline Protein & Function & EV subtype & $\begin{array}{l}\text { RNAi phenotype in } \\
\text { mammalian cells }\end{array}$ & $\begin{array}{l}\text { Present in } \\
\text { Helminth EVs }\end{array}$ & $\begin{array}{l}\text { RNAi phenotypes of } \\
\text { C. elegans orthologues }\end{array}$ & Refs \\
\hline HRS/HGS & ESCRT-0 component & Exo & EV secretion $\downarrow$ & - & Gro, Dpy, Unc & {$[36]$} \\
\hline STAM & ESCRT-0 component & Exo & EV secretion $\downarrow$ & - & Emb, Lva, Let, Ste, Gro, Stp & {$[36]$} \\
\hline TSG101 & ESCRT-I component & Exo \& MV & EV secretion $\downarrow$ & $\mathrm{Fh}^{\mathrm{d}}$ & Emb, Lva, Let, Ste, Gro, Stp & {$[36,37]$} \\
\hline VPS22/SNF8 & ESCRT-II component & Exo & EV secretion $\downarrow$ & - & Emb, Lva, Let, Ste, Gro, Stp & [37] \\
\hline CHMP2A & ESCRT-III component & Exo & EV secretion $\downarrow$ & $\mathrm{Fh}$ & Emb, Lva, Let, Ste, Gro, Stp & {$[37]$} \\
\hline CHMP4 & ESCRT-III component & Exo & EV secretion $\uparrow \downarrow$ & - & Emb, Let, Bmd, Prl & {$[36,37]$} \\
\hline VPS4 & EV abscission & Exo \& MV & EV secretion $\uparrow \downarrow$ & $\mathrm{Fh}, \mathrm{Sm}$ & Emb, Let, Unc, Prl, Gro & {$[36,37]$} \\
\hline ALIX/PDCD6IP & ILV formation/cargo sorting & Exo & EV secretion $\downarrow$ & Fh, Sm, Dd, Hp & Emb, Lva, Let, Ste, Gro, Stp & {$[36,37]$} \\
\hline Syndecan & ILV formation/cargo sorting & Exo & EV secretion $\downarrow$ & - & Let & {$[37]$} \\
\hline Syntenin & ILV formation/cargo sorting & Exo & EV secretion $\downarrow$ & $\mathrm{Fh}, \mathrm{Ov}, \mathrm{Sm}$ & - & [37] \\
\hline Sphingomyelinase & $\begin{array}{l}\text { Ceramide-dependent ILV } \\
\text { formation }\end{array}$ & Exo \& MV & EV secretion $\downarrow^{\mathrm{b}}$ & Fh & Emb, Lva, Let, Ste, Gro, Stp & {$[17,38]$} \\
\hline CD63 antigen & ILV formation/cargo sorting & Exo & No effect & $\mathrm{Fh}, \mathrm{Ov}, \mathrm{Sm}, \mathrm{Sj}$ & - & [37] \\
\hline Rab27 & Fusion of MVB with the PM & Exo & EV secretion $\downarrow$ & $\mathrm{Fh}$ & Emb, Lva, Let, Ste, Gro, Stp & {$[21]$} \\
\hline Rab35 & Fusion of MVB with the PM & Exo & EV secretion $\downarrow$ & $\mathrm{Fh}$ & Let & {$[39]$} \\
\hline Rab11 & Fusion of MVB with the PM & Exo & EV secretion $\downarrow$ & $\mathrm{Fh}, \mathrm{Sm}, \mathrm{Sj}, \mathrm{Hp}$ & Emb, Lva, Let, Ste, Gro, Stp & {$[40,41]$} \\
\hline Ral-1/Ral-A & Fusion of MVB with the PM & Exo & EV secretion $\downarrow$ & $\mathrm{Fh}, \mathrm{Hp}$ & Let & {$[22]$} \\
\hline Syntaxin & Q-SNARE & Exo & EV secretion $\downarrow$ & $\mathrm{Fh}, \mathrm{Sm}$ & Let & {$[42]$} \\
\hline SNAP-25 & T-SNARE & Exo & ND & $\mathrm{Fh}, \mathrm{Sm}$ & Emb, Lva, Let, Ste, Gro, Stp & {$[8]$} \\
\hline YKT6 & R-SNARE & Exo & EV secretion $\downarrow$ & - & Clr, Emb, Sck, Gro, Stp & {$[43]$} \\
\hline $\begin{array}{l}\text { Phospholipid } \\
\text { translocases }^{\text {a }}\end{array}$ & PM curvature & MV & ND & $\mathrm{Fh}$ & $\begin{array}{l}\text { Emb, Lva, Let, Ste, Gro, Stp, } \\
\text { Spn }\end{array}$ & {$[25]$} \\
\hline
\end{tabular}




\begin{tabular}{|c|c|c|c|c|c|c|}
\hline Calpain & Cytoskeletal remodelling & MV & ND & $\mathrm{Fh}, \mathrm{Ov}, \mathrm{Sm}$ & Emb, Lva, Let, Ste, Gro, Stp & [25] \\
\hline Phospholipases & $\begin{array}{l}\text { Signal-induced cytoskeletal } \\
\text { regulation }\end{array}$ & Exo \& MV & EV secretion $\downarrow$ & $\mathrm{Fh}$ & Emb, Lva, Let, Ste, Gro, Stp & [44] \\
\hline ARF6 & $\begin{array}{l}\text { Reorganization of the actin } \\
\text { cytoskeleton }\end{array}$ & Exo \& MV & EV secretion $\downarrow$ & $\mathrm{Fh}, \mathrm{Sj}$ & Emb, Lva, Let, Ste, Gro, Stp & [44] \\
\hline vATPase (V0) & Fusion of MVB with the PM & Exo & MVB accumulation $^{c}$ & $\mathrm{Fh}, \mathrm{Sj}, \mathrm{Hp}, \mathrm{Bm}$ & Clr, Emb, Sck, Gro, Stp & [45] \\
\hline
\end{tabular}

${ }^{\mathrm{a}}$ flippases, floppases and scramblases; ${ }^{\mathrm{b}}$ phenotype observed using chemical inhibitors; ${ }^{\mathrm{c}}$ in C. elegans. ${ }^{\mathrm{d}}$ Abbreviations: Fh, Fasciola hepatica; Ov, Opisthorchis viverrini; Sm, Schistosoma mansoni; Sj, Schistosoma japonicum; Dd, Dicrocelium dendriticum; Hp, Heligsomoides polygyrus; Bm, Brugia malayi; PM, plasma membrane; Exo, exosome, MV, microvesicle. Ste, sterile; Lva, larval arrest; Emb, embryonic lethal; Gro, slow growth; Unc, locomotion abnormal; Dpy, dumpy; Clr, clear; Stp, sterile progeny; Bmd, organism morphology abnormal; Let, larval lethal; Prl, paralysed; Sck, sick; Spn, Abnormal spindle orientation; ND, not determined. 


\section{Trends}

- Parasite-derived EVs are now recognised as important mediators of molecular communication between host and parasite.

- Transcriptomics and proteomics profiling has identified a range of immunomodulatory cargo molecules packaged into these but has also begun to shed light on the mechanisms used by helminths to generate and release EVs into the host microenvironment.

- Although technically challenging, selective inhibition of parasite EV biogenesis pathways would prevent the delivery of a range of immunomodulators to host cells and tip the immune balance in favour of parasite elimination. 


\section{Outstanding questions}

- What mechanisms/pathways are used by helminths to produce and export EVs?

- Are these pathways novel or distinct from those used by mammalian cells?

- Are helminth EVs secreted constitutively or in response to stress?

- Do drug-induced MVs/blebs contribute to mechanisms of drug resistance in parasites?

- Are parasite-derived EVs essential for survival in the mammalian host?

- Can selective inhibitors of key "checkpoints" in parasite EV biogenesis pathways be developed? 


\section{Figure 1}
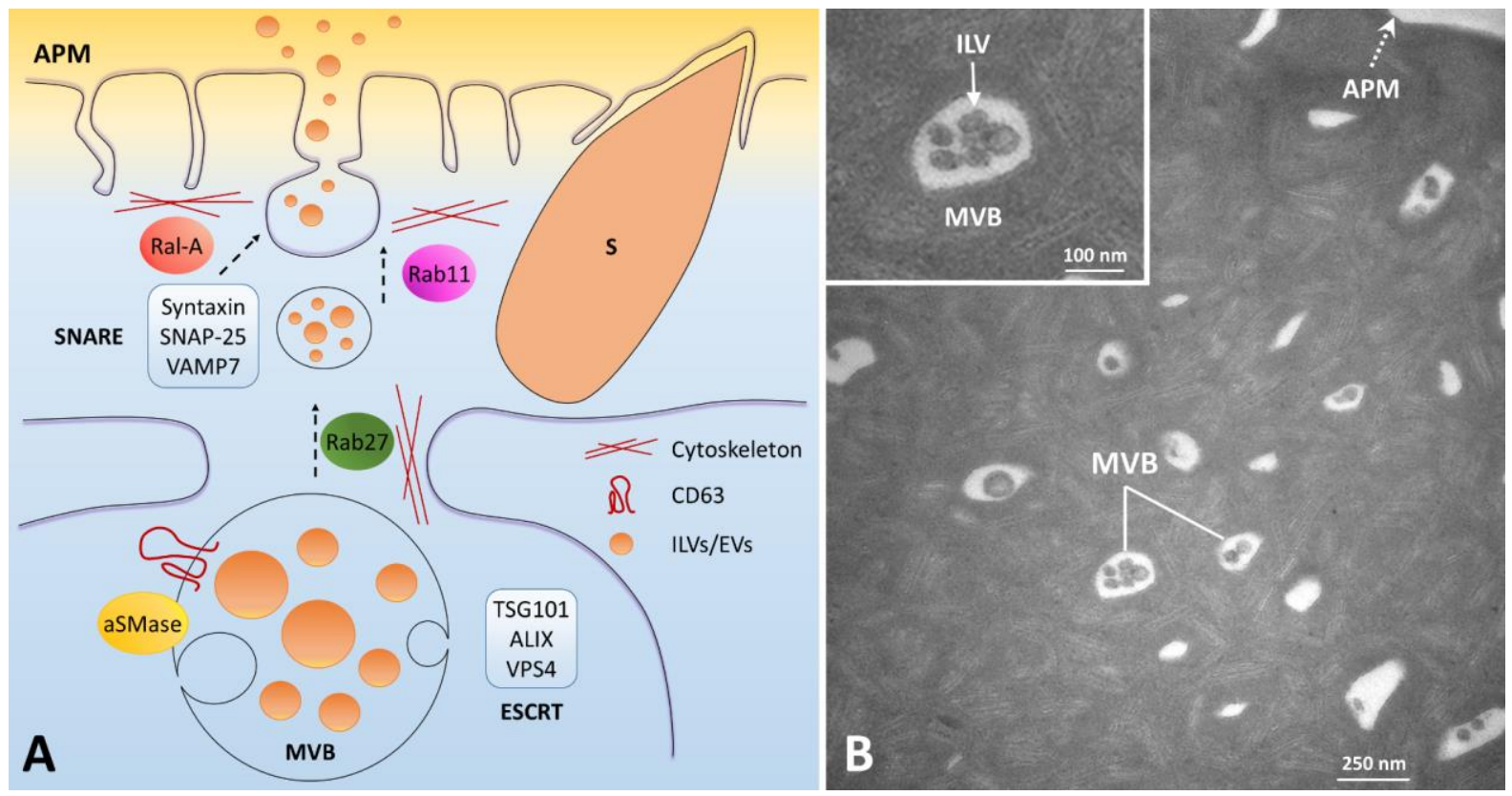

Figure 2

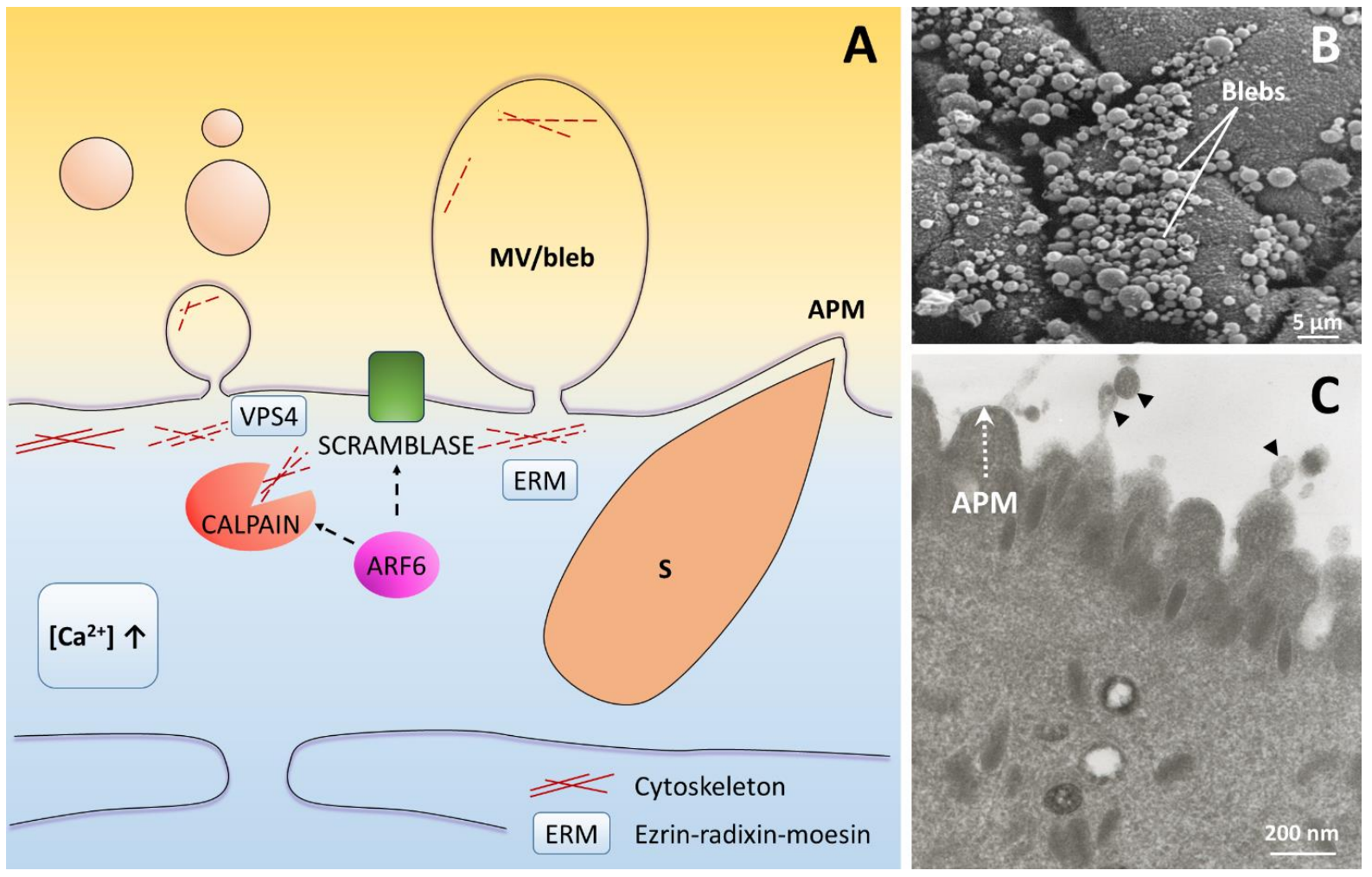

